\title{
Concatenated Space-Time Block Coding With Trellis Coded Modulation in Fading Channels
}

\author{
Yi Gong, Student Member, IEEE, and Khaled Ben Letaief, Senior Member, IEEE
}

\begin{abstract}
Trellis coded modulation (TCM) is a bandwidth efficient transmission scheme that can achieve high coding gain by integrating coding and modulation. This paper presents an analytical expression for the error event probability of concatenated space-time block coding with TCM which reveals some dominant factors affecting the system performance over slow fading channels when perfect interleavers are used. This leads to establishing the design criteria for constructing optimal trellis codes of such concatenated system over slow flat fading channels. Through simulation, significant performance improvement is shown to be obtained by concatenating the interleaved streams of these codes with space-time block codes over fading channels. Simulation results also demonstrate that these trellis codes have better error performance than traditional codes designed for single-antenna Gaussian or fading channels. Performance results over quasi-static fading channels without interleaving are also compared in this paper. Furthermore, it is shown that concatenated space-time block coding with TCM (with/without interleaving) outperforms space-time trellis codes under the same spectral efficiency, trellis complexity, and signal constellation.
\end{abstract}

Index Terms-Fading channels, interleaving, space-time block coding, space-time trellis coding, trellis coded modulation, wireless communications.

\section{INTRODUCTION}

$\mathbf{T}$ HE PERFORMANCE of wireless communications is severely degraded by signal fading caused by multipath propagation and interference from other users [1], [2]. Fortunately, the effects of fading can be substantially mitigated by the use of diversity. Antenna diversity where sufficiently separated or different polarized multiple antennas are put at either the receiver, the transmitter, or both, is being widely considered. In light of prior and current comprehensive research work on signal processing at the receiver end [3]-[5], receive antenna diversity is well understood and widely exploited. However, receive antenna diversity is impractical in applications such as broadcasting or forward link transmission in wireless cellular systems for obvious reasons. In such situations, transmit antenna diversity becomes more and more attractive.

As demonstrated by Foschini and Gans [6], the theoretical capacity over multiple-input-multiple-output (MIMO)

\footnotetext{
Manuscript received November 6, 2000; revised December 13, 2001; accepted December 21, 2001. The editor coordinating the review of this paper and approving it for publication is J. V. Krogmeier. This work was supported in part by the Hong Kong Telecom Institute of Information Technology and the Hong Kong Research Grant Council under Grant HKUST7030/01. This work was presented in part at the IEEE Wireless Communications and Networking Conference, WCNC'00, Chicago, IL, September 2000.

The authors are with the Department of Electrical and Electronic Engineering, Hong Kong University of Science and Technology, Clear Water Bay, Hong Kong (e-mail: eegy@ee.ust.hk; eekhaled@ee.ust.hk).

Digital Object Identifier 10.1109/TWC.2002.804180
}

Rayleigh fading channels increases linearly with $\min (N, M)$ if the fading coefficients between any pair of $N$ transmit antennas and $M$ receive antennas are statistically independent and known to the receiver. In order to optimally exploit this type of diversity and approach Foschini's capacity bound, many transmit diversity schemes have been recently proposed, which can be classified into three major categories: layered space-time (LST) architecture [7], space-time trellis coding [8], [9], and space-time block coding [10], [11]. Throughout this paper, we only consider the full-diversity order coding schemes, which are space-time trellis coding and space-time block coding.

By efficiently integrating the spatial domain and temporal domain, space-time trellis coding achieves a full diversity of order $N M$ and substantial coding gain. The disadvantage of this approach is that when the number of antennas is fixed, the decoding complexity (measured by the number of trellis states at the decoder) grows exponentially as a function of both bandwidth efficiency and diversity order. Thus, space-time codes with low decoding complexity while retaining acceptable performance are quite desirable. In [10], Alamouti proposed a remarkable 2-transmit-antenna diversity scheme as a solution. This approach has a very simple decoding process while retaining the full diversity gain $2 M$. It was later generalized to an arbitrary number of transmit antennas as space-time block coding [11]. Since space-time block coding provides a simple way to upgrade current wireless systems to multiple transmit antenna systems while keeping a full diversity gain, it promises to be an alternative and attractive solution for high data rate wireless communications. Space-time block codes (STBCs), however, are not designed to provide significant coding gain. Hence, powerful outer codes can be concatenated with space-time block coding to have a required coding gain. It was shown in [12], that optimal trellis codes designed for the additive white Gaussian noise (AWGN) are also the best codes that have optimal error event probability for concatenation with STBCs over Rayleigh fading channels. However, detailed simulation results of this scheme have not been provided. Furthermore, the conclusion therein are based on the assumption that the channel fading is constant during a frame and independent between frames and this may not be applicable in many situations such as fast fading. Since interleaving can combat slow fading effectively, it is also necessary to find optimal outer trellis codes over slow fading when interleavers are used.

In this paper, a system concatenating STBC with TCM over fading channels is proposed and analyzed. We present an analytical expression for the error event probability of the 
proposed system which reveals some dominant factors such as the product-sum distance and time diversity that affect the system performance when perfect interleavers are used. This leads to establishing the design criteria of optimal trellis codes for use with space-time block coding over flat fading channels. These results also apply to fast fading channels where the maximum Doppler spread normalized by the symbol rate is of the order of $10^{-2}$. Based on these criteria, some optimal trellis codes concatenated with space-time block coding are constructed. Through simulation, significant performance improvement is shown to be achieved by concatenating the interleaved streams of these codes with space-time block coding over fading channels. Simulation results demonstrate that the proposed outer trellis codes have better error performance than those codes designed for single-antenna Gaussian or fading channels when concatenated with space-time block coding. Performance results of this concatenated scheme using multiple receive antennas over quasi-static fading without the use of interleavers are also given for comparison. It is shown that interleaving significantly improves the system performance in terms of frame error rate (FER). In particular, the proposed scheme (with/without interleaving) has better performance than some space-time trellis codes (STTCs) under the same spectral efficiency, signal constellation, and trellis complexity.

The rest of this paper is organized as follows. Section II briefly describes a space-time coded system concatenating STBC and TCM. In Section III, an upper bound on the error event probability is derived under the assumption of perfect interleaving. Furthermore, the design criteria for constructing trellis codes that are optimal for use with space-time block coding over interleaved flat fading channels are established. Based on these criteria, some 8-PSK trellis codes are constructed in Section IV. Numerical results with/without interleaving are provided in Section V. Finally, we draw our conclusions in Section VI.

\section{System Model With TRANSMIT Diversity}

Consider a concatenated space-time coded system with $N$ transmit and $M$ receive antennas which is shown in Fig. 1. The information data is first encoded by the TCM encoder, then interleaved and fed into the space-time block encoder. At each time slot, the output symbols are modulated and transmitted simultaneously each from a different transmit antenna. At the receiver end, the space-time block decoder and deinterleaver followed by Viterbi decoder can be used to decode the received signals. The proposed system model is shown in Fig. 1.

The input bits are continuously entered into the TCM encoder which generates complex constellation symbols fed into the space-time block encoder. In this paper, we consider the transmission matrix $\mathcal{G}$ of the STBC proposed by Alamouti in [10], where $\mathcal{G}$ is given by

$$
\mathcal{G}=\left(\begin{array}{cc}
x_{1} & x_{2} \\
-x_{2}^{*} & x_{1}^{*}
\end{array}\right) .
$$

All symbols in the same row of $\mathcal{G}$ are transmitted simultaneously from two different transmit antennas and all symbols in the same column of $\mathcal{G}$ are transmitted from the same antenna in successive symbol periods. For simplicity, we also set $M=1$

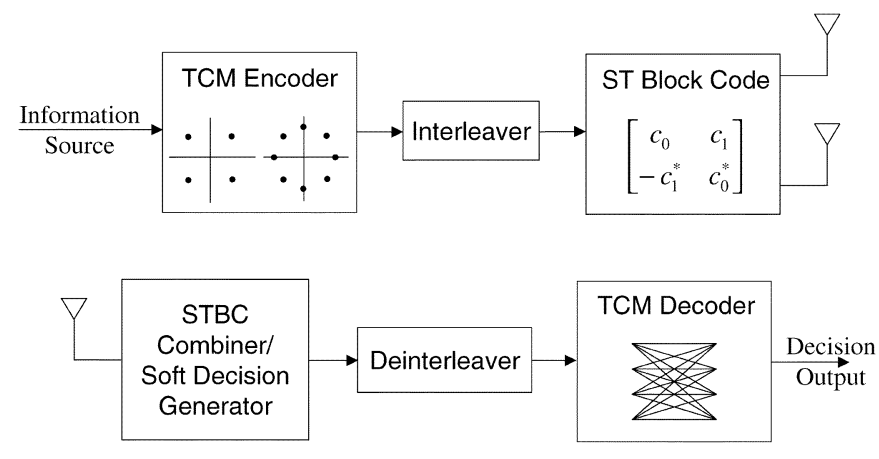

Fig. 1. Block diagram of a space-time coded system concatenating STBC and TCM, $N=2, M=1$.

for performance analysis throughout this paper. Generalization to more than one receive antennas is straightforward and performance results with more than one receive antenna are presented in Section V.

We begin by briefly reviewing the results of Alamouti's scheme. At a given symbol period, the signal transmitted from the first antenna is $c_{0}$ and the signal transmitted from the second antenna is $c_{1}$. In the next symbol period, the signal $-c_{1}^{*}$ is transmitted from the first antenna and the signal $c_{0}^{*}$ is transmitted from the second antenna. Assuming that the fading remains fixed over a duration of length $2 T$, where $T$ is the symbol duration, the received signals can then be expressed as

$$
\begin{gathered}
r_{0} \triangleq r(t)=h_{0} c_{0}+h_{1} c_{1}+n_{0} \\
r_{1} \triangleq r(t+T)=-h_{0} c_{1}^{*}+h_{1} c_{0}^{*}+n_{1}
\end{gathered}
$$

where $h_{n}(n=0,1)$ is the channel gain from the $n+1$ th transmit antenna to the receive antenna and is modeled as independent samples of a zero-mean complex Gaussian random variable with variance $1 / 2$ per dimension. Likewise, $n_{0}$ and $n_{1}$ represent the additive Gaussian noises across the consecutive two symbols and are modeled as complex Gaussian random variables with zero mean and power spectral density $N_{0} / 2$ per dimension. Throughout this paper, we assume that the receiver has perfect channel state information (CSI), then the received signals can be combined in the following two equations to generate soft estimates:

$$
\begin{aligned}
& \tilde{c}_{0}=h_{0}^{*} r_{0}+h_{1} r_{1}^{*}=\left(\left|h_{0}\right|^{2}+\left|h_{1}\right|^{2}\right) c_{0}+h_{0}^{*} n_{0}+h_{1} n_{1}^{*} \\
& \tilde{c}_{1}=h_{1}^{*} r_{0}-h_{0} r_{1}^{*}=\left(\left|h_{0}\right|^{2}+\left|h_{1}\right|^{2}\right) c_{1}+h_{1}^{*} n_{0}-h_{0} n_{1}^{*} .
\end{aligned}
$$

These combined signals are then sent to the soft decision Viterbi decoder for each of the signals $c_{0}$ and $c_{1}$. The Viterbi decoder calculates the metric $m\left(\tilde{c}_{0}, c_{i}\right)$ for the hypothesized branch symbol $c_{i}$ corresponding to the first transmitted symbol $c_{0}$. In this case

$$
m\left(\tilde{c}_{0}, c_{i}\right)=\left(\left|h_{0}\right|^{2}+\left|h_{1}\right|^{2}-1\right)\left|c_{i}\right|^{2}+d^{2}\left(\tilde{c}_{0}, c_{i}\right)
$$

where $d^{2}(x, y)$ is the squared Euclidean distance between signals $x$ and $y$. That is, $d^{2}(x, y)=(x-y)\left(x^{*}-y^{*}\right)$. Similarly, the branch metric for the second transmitted symbol $c_{1}$ can be obtained. 


\section{PeRformance AnAlysis}

\section{A. Quasi-Static Fading}

We begin by giving the performance analysis and design criterion in quasi-static fading, where the fading coefficients are constant during a frame of length $2 L$ and vary from one frame to another.

Assume that the symbol sequence

$$
\begin{aligned}
\mathbf{C}=\left\{c_{0}, c_{1},-c_{1}^{*}, c_{0}^{*}, c_{2}, c_{3},\right. \\
\left.\quad-c_{3}^{*}, c_{2}^{*}, \ldots, c_{2 L-2}, c_{2 L-1},-c_{2 L-1}^{*}, c_{2 L-2}^{*}\right\}
\end{aligned}
$$

is transmitted. The maximum-likelihood decoder decides in favor of the coded sequence

$$
\begin{aligned}
\mathbf{E}=\left\{e_{0}, e_{1},-e_{1}^{*}, e_{0}^{*}, e_{2}, e_{3},\right. & \\
& \left.-e_{3}^{*}, e_{2}^{*}, \ldots, e_{2 L-2}, e_{2 L-1},-e_{2 L-1}^{*}, e_{2 L-2}^{*}\right\}
\end{aligned}
$$

if $m\left(\mathbf{R}, \mathbf{E} ; h_{0}, h_{1}\right) \geq m\left(\mathbf{R}, \mathbf{C} ; h_{0}, h_{1}\right)$ where $\mathbf{R}$ is the received signal sequence and $m\left(\mathbf{R}, \mathbf{E} ; h_{0}, h_{1}\right)$ is the decoding metric related to E. Similarly, $m\left(\mathbf{R}, \mathbf{C} ; h_{0}, h_{1}\right)$ is the decoding metric related to $\mathbf{C}$. Thus

$$
\begin{aligned}
P\left(\mathbf{C} \rightarrow \mathbf{E} \mid h_{0}, h_{1}\right)=P[ & m\left(\mathbf{R}, \mathbf{E} ; h_{0}, h_{1}\right) \\
& \left.\geq m\left(\mathbf{R}, \mathbf{C} ; h_{0}, h_{1}\right) \mid h_{0}, h_{1}\right] .
\end{aligned}
$$

When fading is constant during a frame and varies from one frame to another independently, then by using the Chernoff bound [13] the pairwise error probability can be shown to be upper bounded by [12]

$$
\begin{aligned}
P(\mathbf{C} \rightarrow \mathbf{E}) & \leq\left(1+\frac{E_{s}}{4 N_{0}} \sum_{i=0}^{2 L-1}\left|c_{i}-e_{i}\right|^{2}\right)^{-2} \\
& \leq\left(\frac{E_{s}}{4 N_{0}} d_{\text {free }}^{2}\right)^{-2} .
\end{aligned}
$$

Thus, the design criterion for the trellis code is the maximization of free distance. Hence, the optimal trellis codes designed for AWGN channels are also optimal for use with space-time block coding in quasi-static fading [12]. It must be noted that the performance bound established above is for the error event probability. Since we cannot relate the error event probability with its transfer function in this case, the bit error probability may not be optimized based on this design criterion.

\section{B. Perfect Interleaving}

In the following, we derive performance analysis and design criteria for the proposed system concatenating STBC with TCM in quasi-static fading channels when perfect 2-symbol-wise interleaving is exploited. Alternatively, this is also applicable to fast fading channels where the maximum Doppler spread normalized by the symbol rate is of the order of $10^{-2} .{ }^{1}$ Since we use Alamouti's scheme, perfect 2-symbol-wise interleaving guarantees that channel fading is constant in a time interval of duration $2 T$, but independently varies from one time interval of duration $2 T$ to another. Let $h_{n i}$ denote the channel gain from the $n$th $(n=1,2)$ transmit antenna to the receive antenna at the $i$ th symbol period, $j=0,1, \ldots, 2 L-1$, which is modeled as independent samples of a zero-mean complex Gaussian random variable with variance $1 / 2$ per dimension. Define $\mathbf{h}_{0}=$ $\left(h_{00}, h_{02}, \ldots, h_{0(2 L-2)}\right)$ and $\mathbf{h}_{1}=\left(h_{10}, h_{12}, \ldots, h_{1(2 L-2)}\right)$. Then, the pairwise error probability, conditioned on the complex channel gains $\mathbf{h}_{0}$ and $\mathbf{h}_{1}$, is well approximated by (6) shown at the bottom of the page, where $2+$ means that the increment of index $i$ is in steps of two.

Since the absolute values of $\mathbf{h}_{0}$ and $\mathbf{h}_{1}$ are independent and Rayleigh distributed, by averaging (6) over the Rayleigh distributed probability density function (pdf) [ $2 L$-fold product], we have

$$
\begin{aligned}
P(\mathbf{C} \rightarrow \mathbf{E}) \leq \prod_{i=0,2+}^{2 L-2}\left[1+\frac{E_{s}}{4 N_{0}}\right. & \left.\cdot\left(\left|c_{i}-e_{i}\right|^{2}+\left|c_{i+1}-e_{i+1}\right|^{2}\right)\right]^{-2} \\
= & \prod_{i \in \eta}\left[1+\frac{E_{s}}{4 N_{0}}\left(\left|c_{i}-e_{i}\right|^{2}+\left|c_{i+1}-e_{i+1}\right|^{2}\right)\right]^{-2}
\end{aligned}
$$

where $\eta$ is the set of all $i$ for which $c_{i} \neq e_{i}$ or $c_{i+1} \neq e_{i+1}$. Denote the number of elements in $\eta$ by $l_{\eta}$. Then, at high signal-tonoise ratios (SNRs), (7) can be expressed as

$$
P(\mathbf{C} \rightarrow \mathbf{E}) \leq \frac{1}{\left[\left(\frac{E_{s}}{4 N_{0}}\right)^{l_{\eta}} d_{P}\left(l_{\eta}\right)\right]^{2}}
$$

where

$$
d_{P}\left(l_{\eta}\right)=\prod_{i \in \eta}\left[\left|c_{i}-e_{i}\right|^{2}+\left|c_{i+1}-e_{i+1}\right|^{2}\right]
$$

is the product of Euclidean distances associated with two consecutive symbols along the error event path $(\mathbf{C} \rightarrow \mathbf{E})$. We may refer to $d_{P}\left(l_{\eta}\right)$ as the product-sum distance over span 2 . In addition, $l_{\eta}$ is referred to as the effective length of this error event over span 2 .

${ }^{1}$ This still satisfies the minimum constant fading property required for decoding STBCs.

$$
\begin{aligned}
P\left(\mathbf{C} \rightarrow \mathbf{E} \mid \mathbf{h}_{0}, \mathbf{h}_{1}\right) & \leq \exp \left\{\sum_{i=0,2, \ldots, 2 L-2}\left[\left|\left(c_{i}-e_{i}\right) h_{0 i}+\left(c_{i+1}-e_{i+1}\right) h_{1 i}\right|^{2}+\left|\left(e_{i+1}^{*}-c_{i+1}^{*}\right) h_{0 i}+\left(c_{i}^{*}-e_{i}^{*}\right) h_{1 i}\right|^{2}\right] \frac{-E_{S}}{4 N_{0}}\right\} \\
& =\exp \left\{-\frac{E_{s}}{4 N_{0}} \sum_{i=0,2+}^{2 L-2}\left\{\left[\left|c_{i}-e_{i}\right|^{2}+\left|c_{i+1}-e_{i+1}\right|^{2}\right]\left[\left|h_{0 i}\right|^{2}+\left|h_{1 i}\right|^{2}\right]\right\}\right\}
\end{aligned}
$$


Let $P_{e}$ denote the error event probability, then by using the union bound an upper bound can be obtained as

$$
P_{e} \leq \sum_{L=1}^{\infty} \sum_{\mathbf{C}} \sum_{\mathbf{E} \neq \mathbf{C}} P(\mathbf{C}) P(\mathbf{C} \rightarrow \mathbf{E})
$$

where $P(\mathbf{C})$ is the a priori probability of transmitting the symbol sequence $\mathbf{C}$ with length $2 L$. By summing over all possible $l_{\eta}$ and all possible $d_{P}\left(l_{\eta}\right)$, the error event probability can be further written as

$$
P_{e} \leq \sum_{l_{\eta}} \sum_{d_{P}\left(l_{\eta}\right)} \Xi\left(l_{\eta}, d_{P}\left(l_{\eta}\right)\right)\left[\left(\frac{E_{s}}{4 N_{0}}\right)^{l_{\eta}} d_{P}\left(l_{\eta}\right)\right]^{-2}
$$

where $\Xi\left(l_{\eta}, d_{P}\left(l_{\eta}\right)\right)$ is the average number of error events having the span-2 effective length $l_{\eta}$ and the product-sum distance $d_{P}\left(l_{\eta}\right)$. Clearly, among all terms in (10) the one with the smallest $l_{\eta}$ and the smallest $d_{P}\left(l_{\eta}\right)$ dominates the error event probability at high SNRs. Denoting $R=\min \left(l_{\eta}\right)$ and $d_{\min }(R)=\min \left(d_{P}(R)\right)$, then the error event probability is asymptotically approximated as

$$
P_{e} \cong \Xi\left(R, d_{\min }(R)\right) \frac{1}{\left(\frac{E_{s}}{4 N_{0}}\right)^{2 R}\left[d_{\min }(R)\right]^{2}} .
$$

From (11), we observe that the error event probability asymptotically varies with $2 R$-power of SNR, so a diversity order of $2 R$ is achieved. We further refer to $R$ as the build-in time diversity or effective length of the concatenated space-time code.

The design criteria, in this case, involves the maximization of both the build-in-time diversity and the minimum product-sum distance of trellis code at high SNRs for Rayleigh fading. This conclusion is, therefore, different from that of conventional TCM where the minimum product distance needs to be maximized. Thus, new optimal codes can be found upon these new criteria.

Next, we look at the case of low SNRs. Equation (7) can be rewritten as

$$
P(\mathbf{C} \rightarrow \mathbf{E}) \leq\left[1+\frac{E_{s}}{4 N_{0}} \sum_{i=0}^{2 L-1}\left|c_{i}-e_{i}\right|^{2}+o\left(\frac{E_{s}}{4 N_{0}}\right)\right]^{-2}
$$

where $o\left(E_{s} / 4 N_{0}\right)$ denotes the summation of all the terms which include higher order quantities of $\left(E_{s} / 4 N_{0}\right)$. Equation (12) indicates that the squared Euclidean distance becomes the main factor. Thus, the dominant factor affecting the performance of trellis coded modulation for use with space-time block coding at low SNRs is the free Euclidean distance rather than the product-sum distance and build-in time diversity.

\section{CODE Design}

Based on the criteria obtained above, optimal trellis codes concatenated with space-time block coding assuming ideal interleaving can be constructed. For example, we first consider the code design rules for the 4 -state rate $2 / 3$ 8-PSK trellis code for use with transmit diversity. It is noted that the build-in time diversity $(R)$ of a 4-state code is limited equal to one and, therefore, optimized in this simplest case. In order to increase the product-sum distance of the code, parallel transitions in

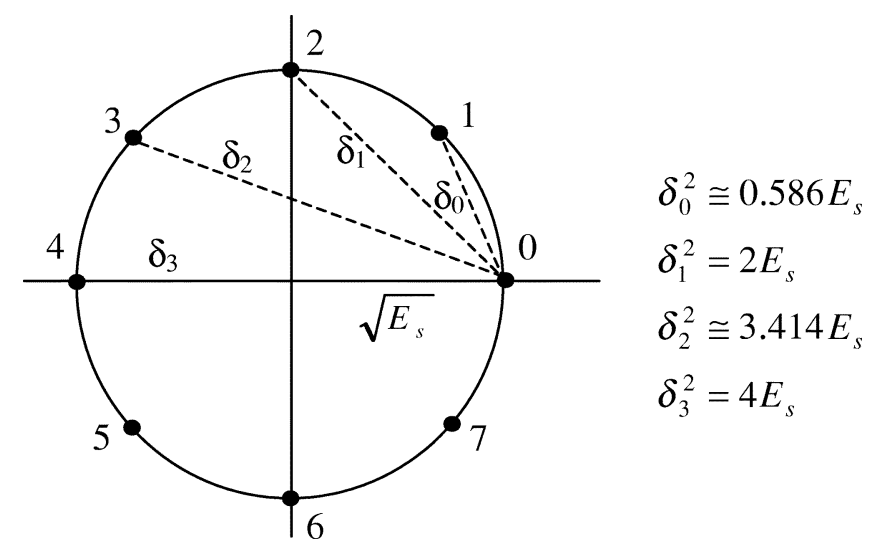

Fig. 2. Eight-PSK signal set.

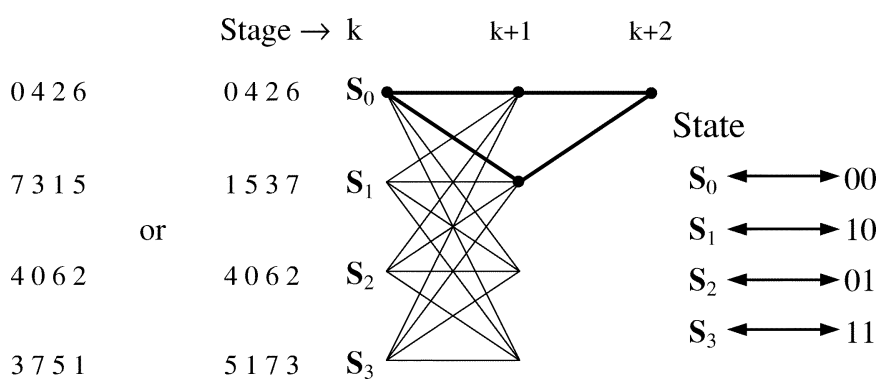

Fig. 3. Trellis diagram of the 4-state 2/3 8-PSK trellis code.

the trellis diagram are avoided. Thus, we can only consider the error events of actual length 2 to maximize the minimum product-sum distance of the underlying code. The signal transitions between states of consecutive stages can be represented by a $4 \times 4$ matrix $\mathbf{G}$, where the $i j$ th element represents the signal transmitted from state $i$ to state $j$ between consecutive stages in the trellis diagram. Using set partitioning, the 8-PSK signal set shown in Fig. 2 is partitioned into two subsets $\mathbf{A}_{0}=(0,2,4,6)$ and $\mathbf{A}_{1}=(1,3,5,7)$. The proposed design rules are given as follows.

Rule 1: Elements of each row of the matrix $\mathbf{G}$ are associated with signals from subsets $\mathbf{A}_{0}$ or $\mathbf{A}_{1}$. Specifically, signals with distance $\delta_{1}$ and $\delta_{3}$ are associated with branches diverging from one state to two adjacent states with a state difference of two and one, respectively, where the state difference is defined as the number of bits in which two states differ (see Fig. 3 for more details).

Rule 2: The distance between branches remerging at one state from two adjacent states with a state difference of two is $\delta_{2}$. The pair of signals remerging from two states with a state difference of one are associated with distance $\delta_{0}$ or $\delta_{3}$.

According to (11), the minimum product-sum distance should be maximized. Rule 1 associates distance $\delta_{1}$ to signals diverging from one state to two adjacent states with a state difference of two (two states with state difference of two are always adjacent) and guarantees that the distance between any two signals diverging from one state is at least $\delta_{1}$. Thus, if we assign $\delta_{2}$ to signals remerging at one state from two states with a state difference of two (Rule 2), the minimum product-sum distance will be $d_{\min }(R)=\min \left(\delta_{1}^{2}+\delta_{2}^{2}, \delta_{0}^{2}+\delta_{3}^{2}\right)=\delta_{0}^{2}+\delta_{3}^{2}=4.586 E_{s}$, which is greater than that of the optimal single-antenna 4-state 


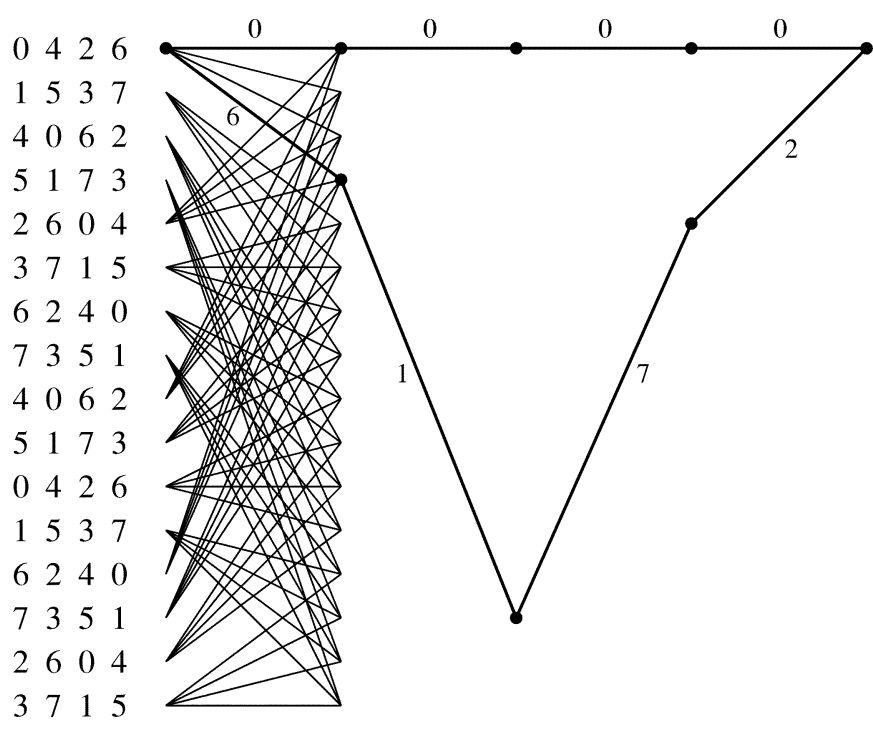

Fig. 4. Trellis diagram of the 16-state 2/3 8-PSK trellis code.

2/3 8-SPK code [14] (referred to as J-L code hereafter) designed for fading channels, whose distance is $\delta_{1}^{2}+\delta_{1}^{2}=4 E_{s}$. Using the above code design rules, we find the best 4 -state $2 / 3$ 8-PSK trellis code for use with transmit diversity when perfect interleavers are assumed. The trellis diagram of this code is shown in Fig. 3. An equivalent code constructed using the design tools is also shown there.

The 8-state trellis code is also constructed. Due to the constraint of the trellis structure, the 8-state code is found to be the Ungerboeck code [15]. Its minimum product-sum distance is $6 E_{s}$. An 8-state code with a larger product-sum distance exists, but it is catastrophic. Obviously, the total diversity $(2 R)$ of both the 4 and 8 -state codes is equal to two, but it will increase to four when the number of states is increased to 16 , since $R$ is increased from one to two. After experimentation with various trellis structures and signal assignments, the 16-state code is also found based on the design criteria, which is shown in Fig. 4. The minimum product-sum distance of this code is equal to $d_{\min }(R)=\left(\delta_{0}^{2}+\delta_{1}^{2}\right)\left(\delta_{0}^{2}+\delta_{1}^{2}\right)=(2+0.586) E_{s} \times(2+$ $0.586) E_{s}=6.69 E_{s}^{2}$. It is verified that the constructed 16-state code has an Ungerboeck representation, which means that it can be generated by a feedback-free convolutional encoder followed by the natural mapping.

\section{Simulation Results}

In this section, some numerical results are presented for the proposed space-time block coding scheme concatenated with TCM with/without interleaving over slow flat Rayleigh fading channels. The proposed system with two transmit antennas and various number of receive antennas is simulated using Monte Carlo simulations, where signals transmitted from different antennas undergo independent flat Rayleigh fading. In this case, these fading coefficients are constant during a frame and vary from one frame to another independently. To allow comparisons with STTCs [8], performance is measured by FER for a frame of 130 symbols and, as mentioned earlier, ideal CSI is assumed at the receiver. In all of the following performance plots, SNR represents the average SNR per receive antenna.

\section{A. Quasi-Static Fading}

In this section, simulation results over quasi-static fading channels without interleaving are provided. It was indicated in [12] that optimal trellis codes designed for the AWGN are also the best codes that have optimal error event probability for concatenation with STBCs over quasi-static Rayleigh fading channels. Thus, we only consider Ungerboeck codes [15] in this case.

Eight- and 16-state rate 2/3 8-PSK Ungerboeck codes concatenated with STBCs are simulated for $M=1, \ldots, 4$ receive antennas over quasi-static flat fading channels. The results are shown in Figs. 5-8. The codes labeled 8-U TCM and 16-U TCM denote the 8-state and 16-state Ungerboeck trellis code, respectively. The performance results of the 4PSK STTCs proposed in [8] and STBC [10] only are also listed for comparisons at a spectral efficiency of $2 \mathrm{~b} / \mathrm{s} / \mathrm{Hz}$ in all these figures. It is interesting to find that the concatenated schemes always have better performance than STTCs at the same number of trellis states. This indicates that the concatenated coding scheme has a larger coding advantage than space-time trellis coding.

A close observation of Fig. 5 indicates that for only one receive antenna, space-time block coding combined with 8 and 16-state Ungerboeck trellis codes has the largest gain over STTCs with 8 and 16 trellis states, respectively. However, the performance gain decreases when the number of receive antennas is increased. This may be due to the fact that STTCs have a better outage capacity than concatenated STBCs when the number of receive antennas is high. Another possible reason is that increasing the constellation size of space-time block coding sacrifices the system performance to some extent. Nevertheless, when the number of receive antennas is less than four, it is concluded that under the same trellis complexity and spectral efficiency, concatenated space-time block coding with TCM achieves better overall performance than STTCs over slow fading channels.

\section{B. Perfect Interleaving}

This section provides simulation results of the proposed STBC system concatenated with TCM over flat fading channels where a 2-symbol-wise interleaver with enough interleaving depth is used. At first, performance comparison of concatenated space-time block coding with the traditional 4-state 8-PSK trellis code designed for single-antenna fading channels (J-L code) and the 4-state 8-PSK code constructed in Section IV is listed in Fig. 9. As predicted by the performance analysis in Section III, our constructed 4-state code has better performance at high SNRs. For instance, the coding advantage at a FER of $10^{-3}$ is about $0.5 \mathrm{~dB}$. The small coding advantage is due to the small increment in product-sum distance. Furthermore, the single-antenna 4-state code optimized for the AWGN channel has worse performance than the two codes mentioned above. This is due to the presence of parallel transitions in the code optimized for the AWGN channel, which limits the effective length to one. For brevity, we avoided including the performance of the conventional 4-state 8-PSK trellis code optimized for the AWGN channel. 


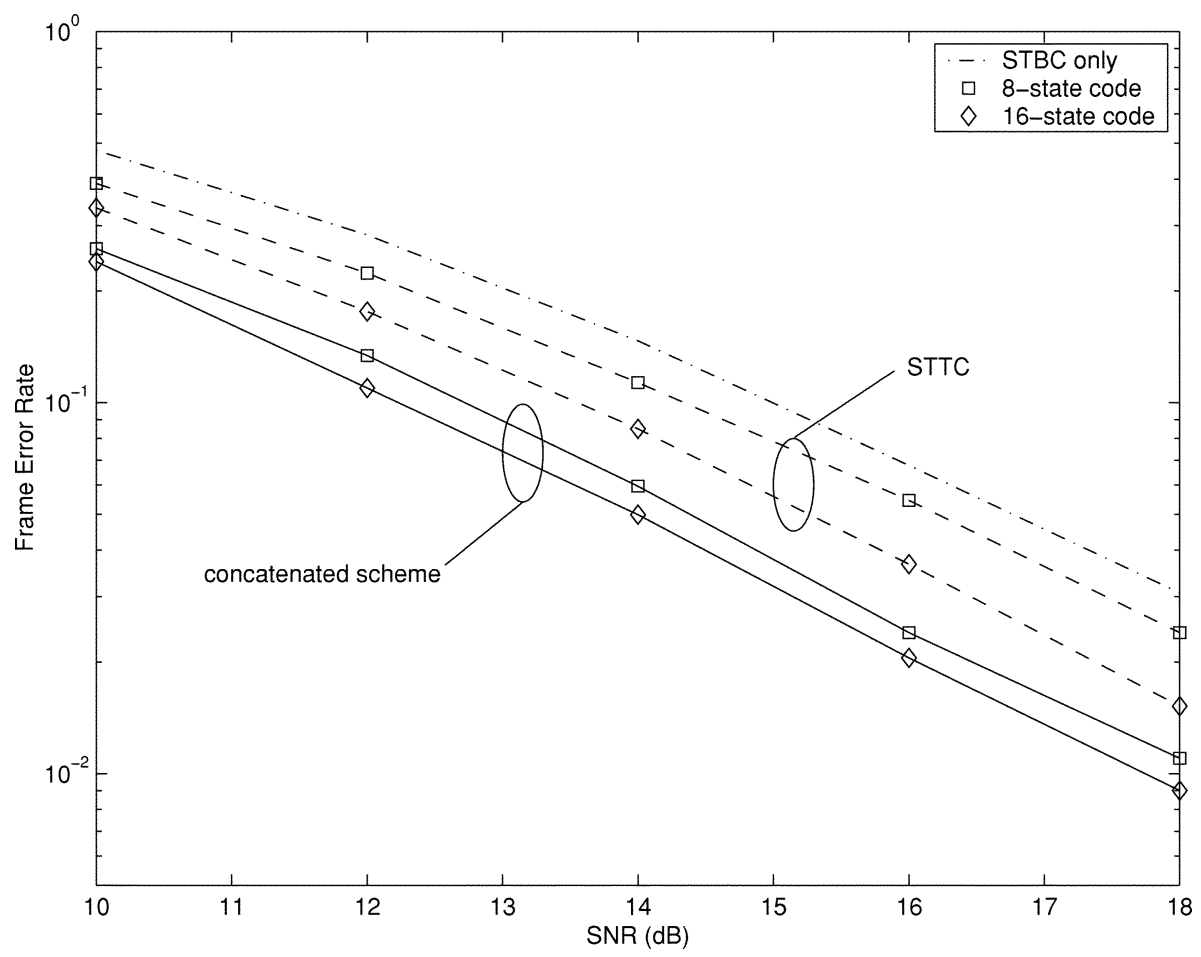

Fig. 5. Quasi-static fading, $M=1$.

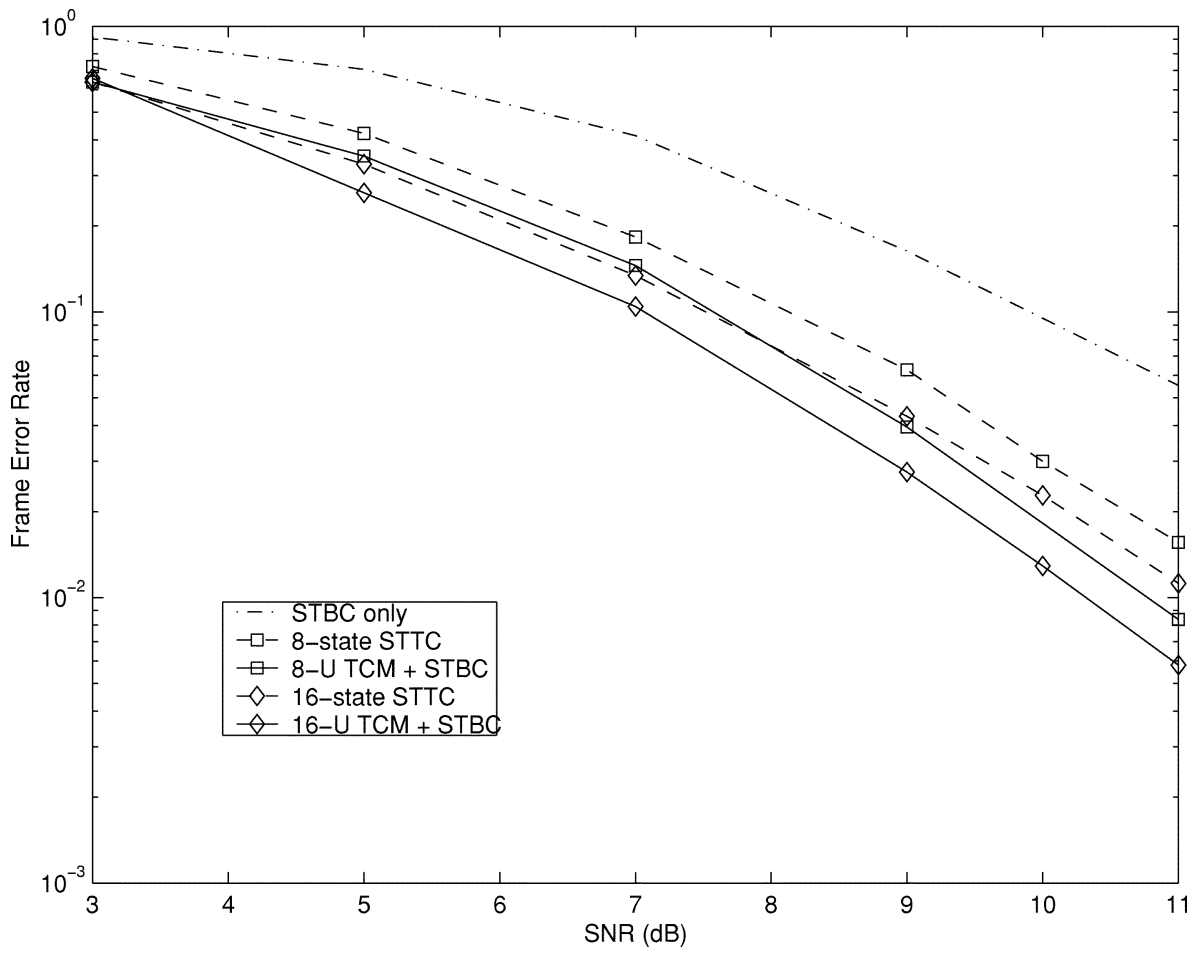

Fig. 6. Quasi-static fading, $M=2$. The dashed line denotes the STTC only and the solid line denotes the concatenated scheme.

Fig. 10 presents performance of our constructed 4, 8, and 16-state trellis codes with STBC transmit diversity using one receive antenna. Recall that for the codes constructed in Section IV, the build-in time diversity of both the 4 and 8 -state codes is equal to two, but it will increase to four as the number of states is increased to 16. According to (8), it is expected that the performance will be improved significantly compared with the 4- and 8-state codes. This is clearly confirmed and shown in Fig. 10.

Performance results of these 4, 8, and 16-state 8-PSK trellis codes are also listed in Figs. 11 and 12, where two and three receive antennas are used, respectively. These figures show that increasing the number of receive antennas reduces the performance gap between trellis codes with different number of states. 


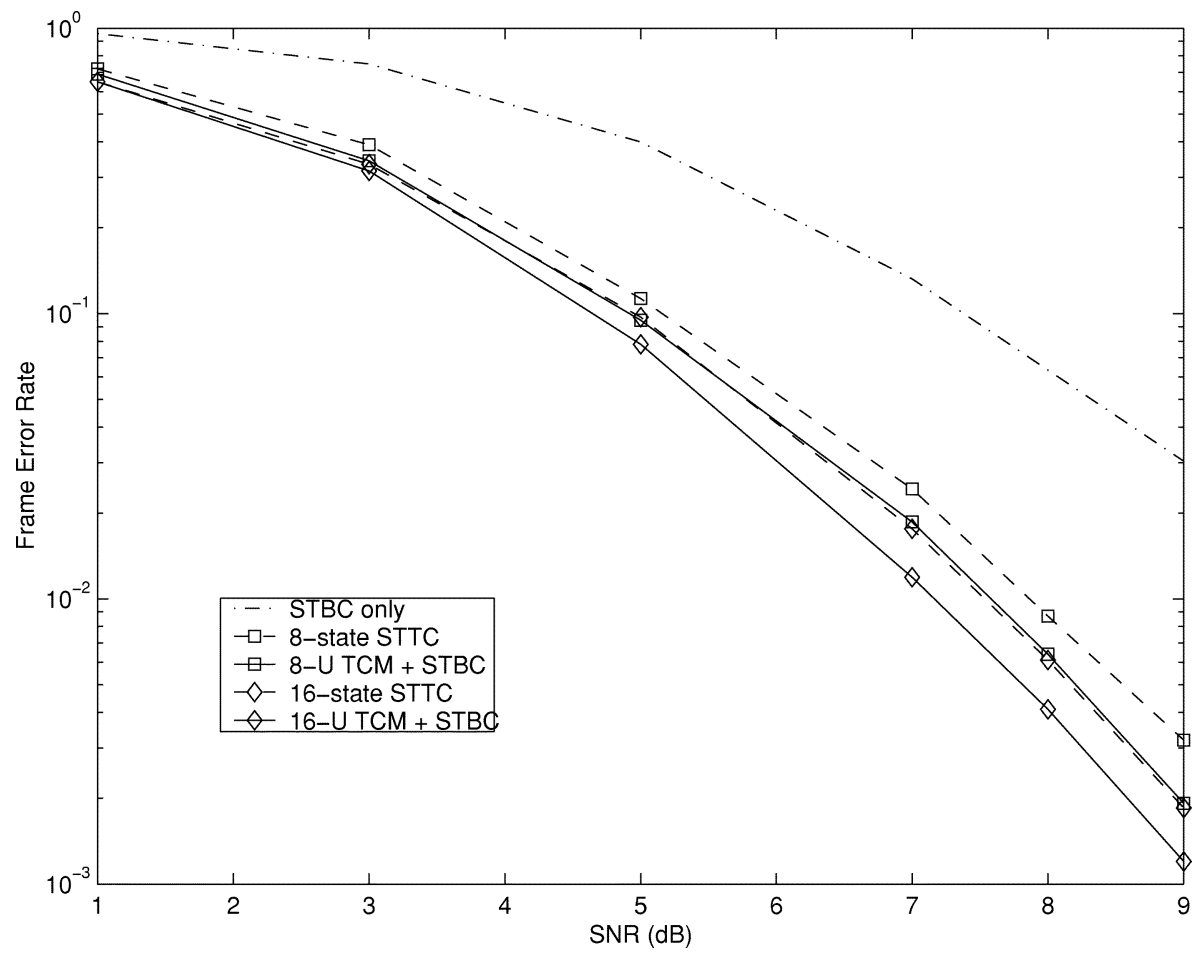

Fig. 7. Quasi-static fading, $M=3$. The dashed line denotes the STTC only and the solid line denotes the concatenated scheme.

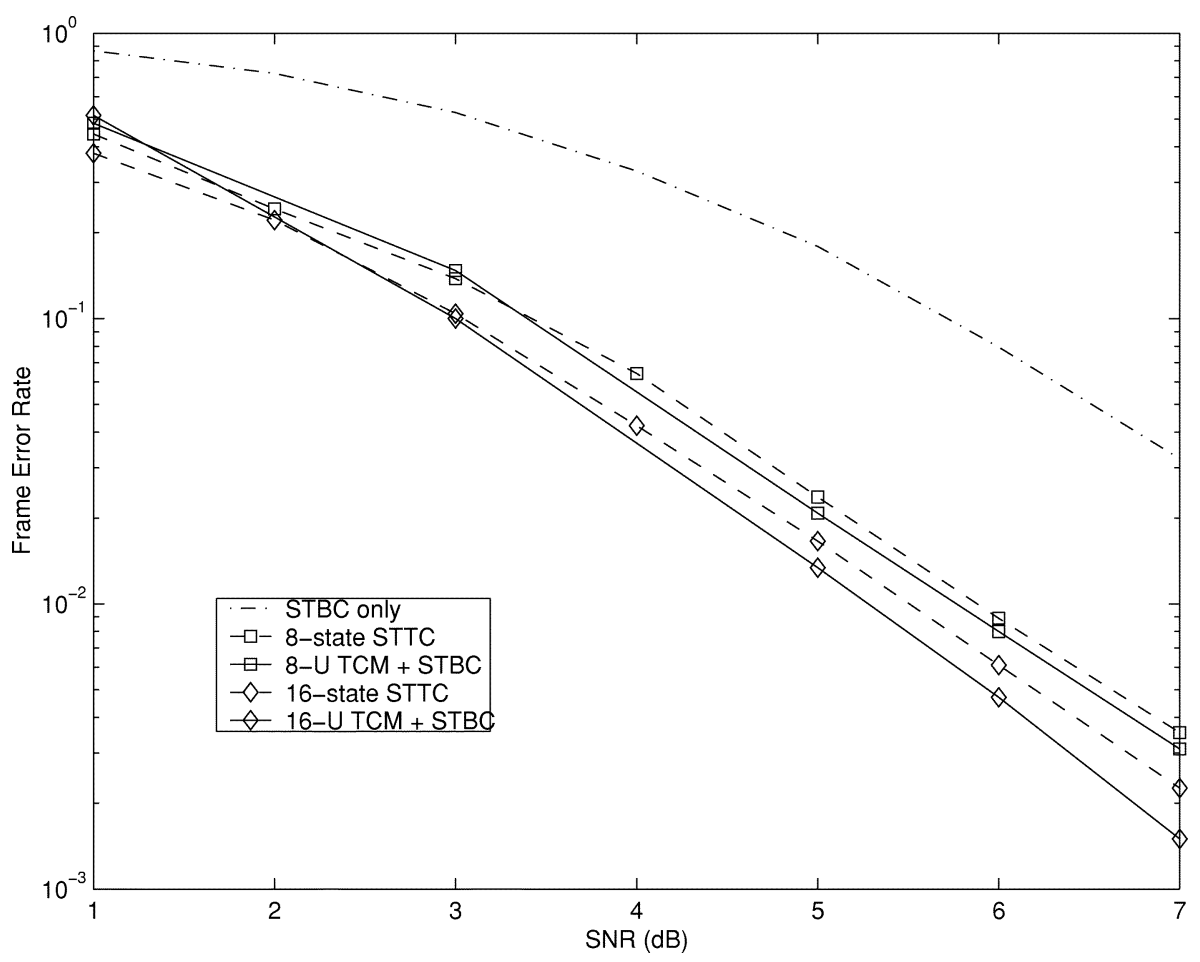

Fig. 8. Quasi-static fading, $M=4$. The dashed line denotes the STTC only and the solid line denotes the concatenated scheme.

Figs. 10-12 also list the system performance without interleaving for comparison, with one, two, and three receive antennas, respectively. For the sake of fair comparisons, only 8 -state and 16-state Ungerboeck trellis codes with/without interleaving are considered (the optimal 4-state code with interleaving is different from that without interleaving). A close observation of these figures reveals that interleaving can cause significant performance improvement compared with the case of without interleaving in quasi-static flat fading channels. For example, it can be seen in Figs. 10-12 that at a FER of $10^{-2}$, about $2-4 \mathrm{~dB}$ improvement can be obtained by the use of interleaving in the concatenated system. Since STTCs are typically designed for slow fading, it can be shown that interleaving them only results in small performance variation. Thus, we did not include performance results of STTCs with interleaving in these plots. As is already shown in Section V-A 


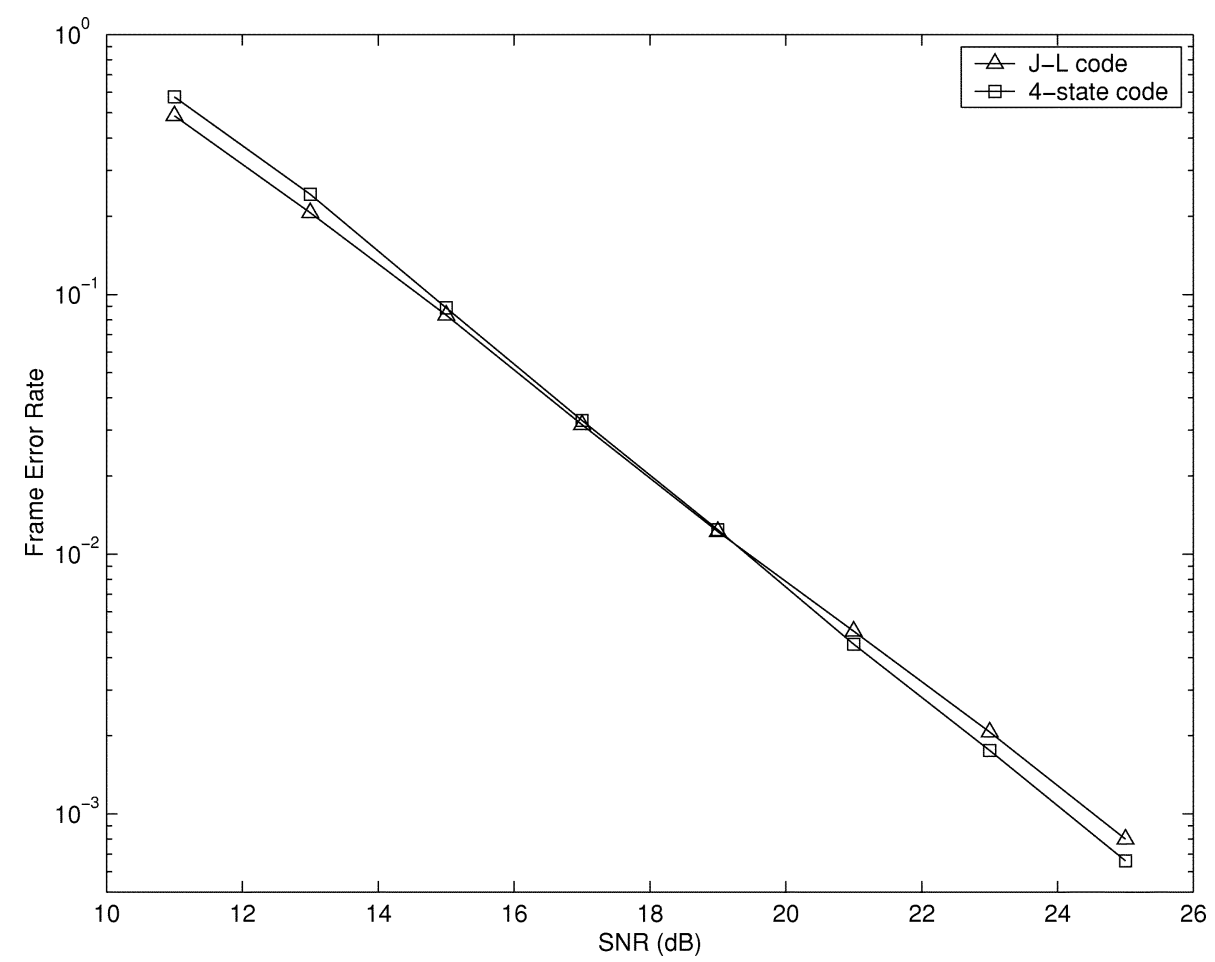

Fig. 9. Concatenating STBC with J-L code and the constructed 4-state code, perfect interleaving, $M=1$.

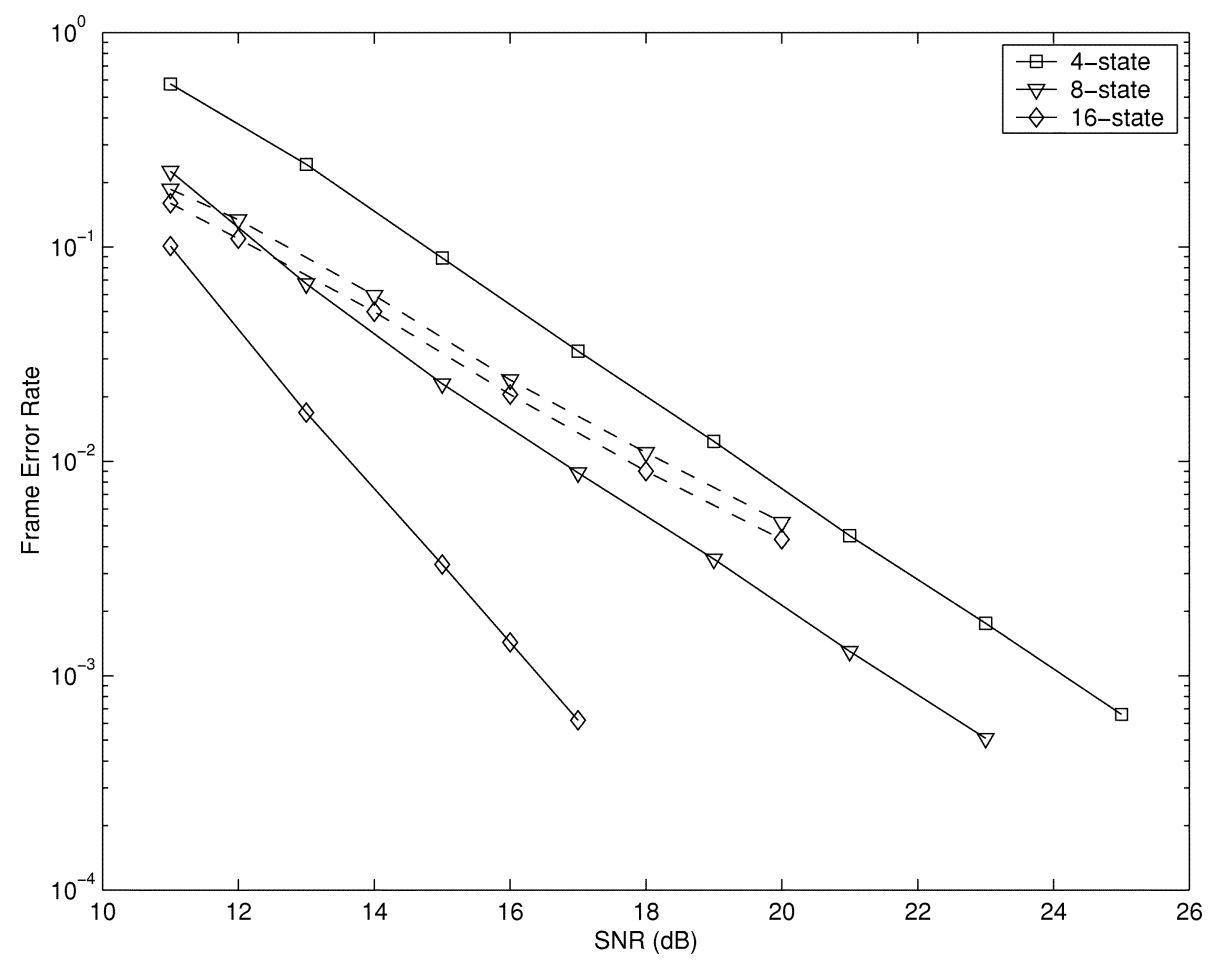

Fig. 10. Perfect interleaving, $M=1$. Performances without interleaving (shown in dashed lines) are also listed for comparison.

that concatenated STBCs have better performance than STTCs in quasi-static fading, the performance gap between them will be much larger in the case of interleaving.

So far we have presented performance of the proposed TCM scheme with STBC transmit diversity and the traditional STTCs with and without interleaving at the same trellis complexity and transmission efficiency of $2 \mathrm{~b} / \mathrm{s} / \mathrm{Hz}$. It is noted that Reed-Solomon (RS) code can be used to increase the error performance by providing more error protection at the expense of reduced spectral efficiency and on the contrary, higher spectral efficiency can be achieved by using higher order signal constellation at the cost of degraded error performance. In order to provide more comparable performance results based on the same signal constellation, spectral efficiency and trellis complexity, an outer $(64,48)$ RS code over $\operatorname{GF}\left(2^{4}\right)$ is concatenated with our proposed 8-state 8-PSK scheme 


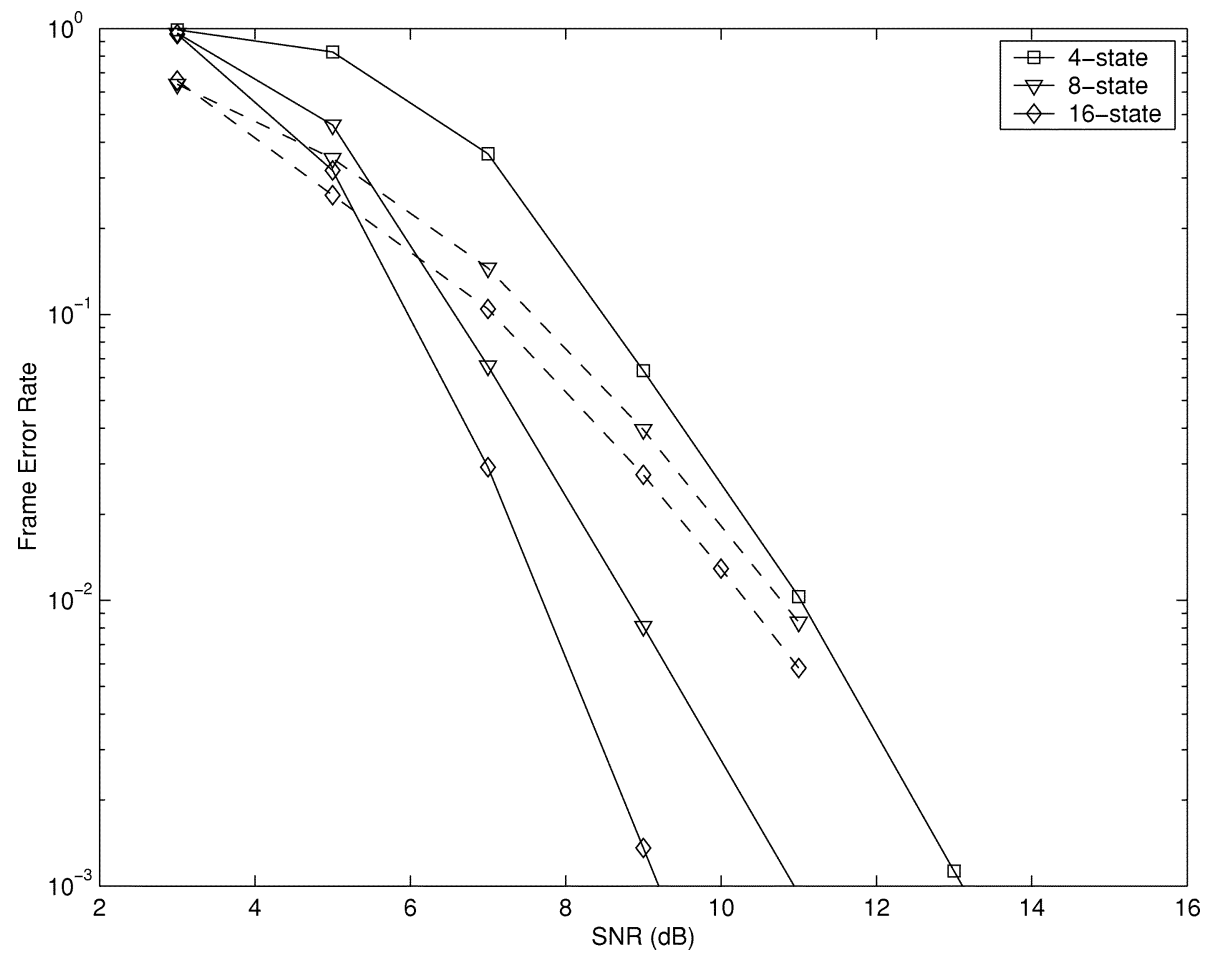

Fig. 11. Perfect interleaving, $M=2$. Performances without interleaving (shown in dashed lines) are also listed for comparison.

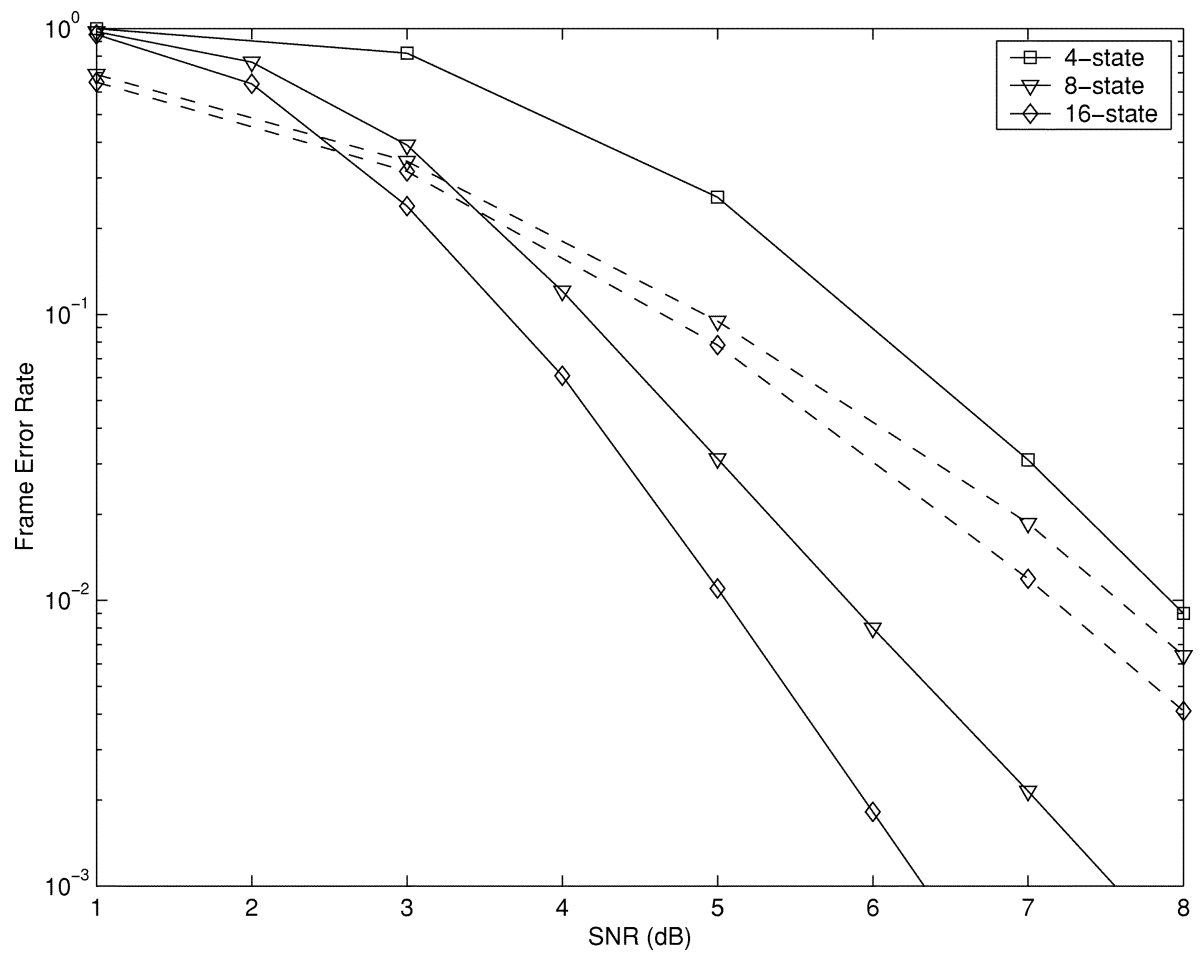

Fig. 12. Perfect interleaving, $M=3$. Performances without interleaving (shown in dashed lines) are also listed for comparison.

to achieve a spectral efficiency of $2 \times 3 / 4=1.5 \mathrm{~b} / \mathrm{s} / \mathrm{Hz}$. Likewise, an outer $(64,32) \mathrm{RS}$ code over $\operatorname{GF}\left(2^{6}\right)$ is employed with the 8-state 8-PSK ST trellis code [8] to have the same spectral efficiency of $3 \times 1 / 2=1.5 \mathrm{~b} / \mathrm{s} / \mathrm{Hz}$. In both of these concatenated schemes to be compared next, the frame length is set to 128. In Fig. 13, we provide performance curves for these two concatenated schemes using two transmit and one receive antennas with/without interleaving. We first focus on the case without interleaving. Compared with Fig. 5, performance of our 8-state scheme increases slightly after employing an outer RS code. The performance improvement is small because the burst of errors caused by the static fading is beyond the error correction capability of the employed RS code. In the case of interleaving, however, these bursts of errors are effectively spread such that the outer RS code is able to efficiently correct them. Accordingly, performances of both our scheme and 


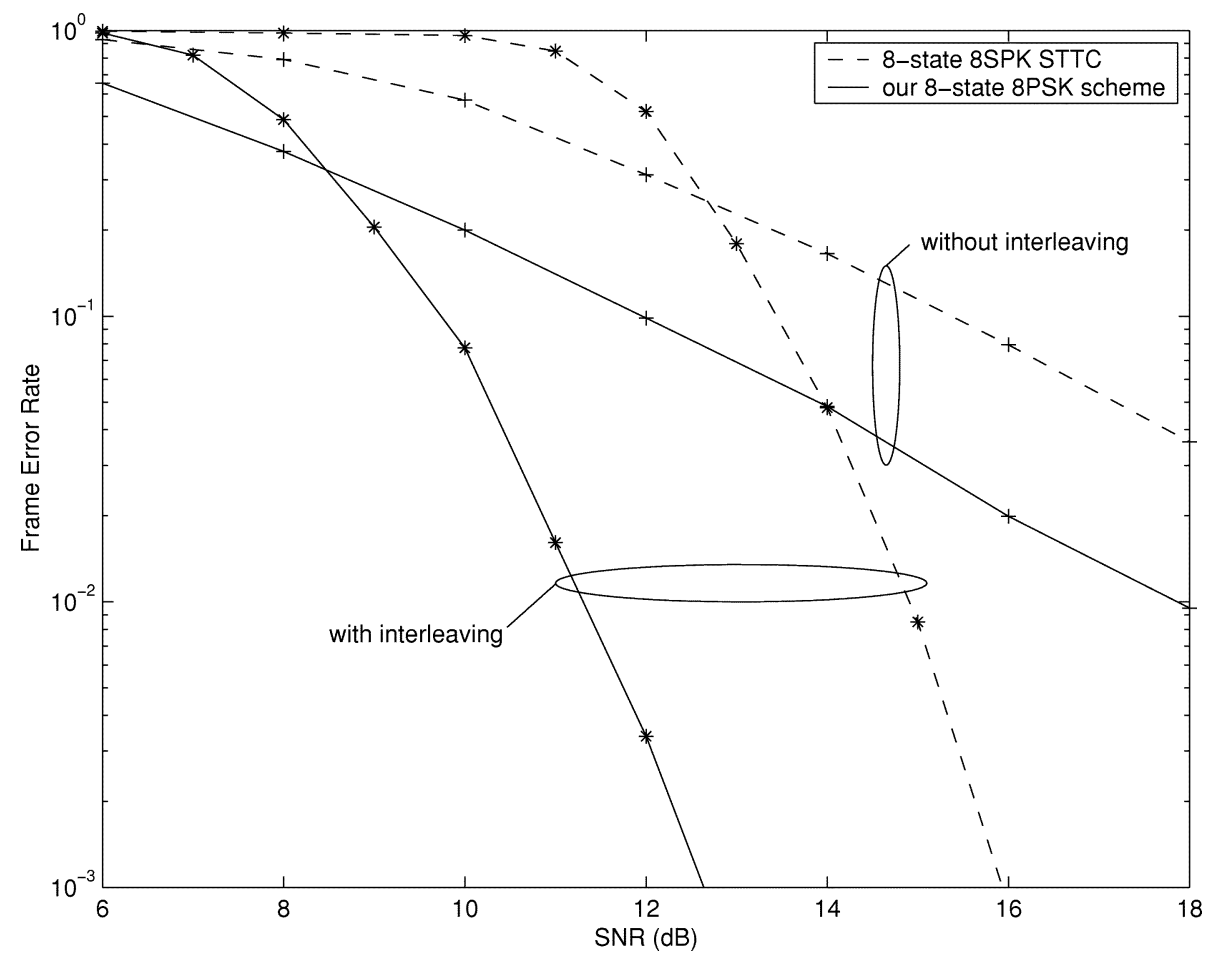

Fig. 13. Our 8-state 8-PSK scheme versus 8-state 8-PSK STTC at a spectral efficiency of $1.5 \mathrm{bit} / \mathrm{sec} / \mathrm{Hz} . M=1$.

STTC are greatly improved. It is observed that, either with interleaving or without interleaving, our scheme is $3-4 \mathrm{~dB}$ better than STTC. Thus, we may conclude that our scheme outperforms STTCs at the same spectral efficiency, signal constellation and trellis complexity over fading channels.

\section{CONCLUSION}

An analytical expression for the error event probability of space-time block coding concatenated with TCM is presented in this paper when perfect interleavers are used. Design criteria for constructing good trellis codes are then established over flat fading channels. Based on these criteria, optimal trellis codes concatenated with space-time block coding are constructed. Through simulation, it is shown that the constructed 8-PSK codes outperform the traditional 8-PSK trellis codes designed for single-antenna Gaussian or fading channels. Performance results over quasi-static fading channels without interleaving are also presented. It is shown that the concatenated STBCs (with/without interleaving) present better performance than STTCs at the same spectral efficiency, signal constellation and trellis complexity.

\section{ACKNOWLEDGMENT}

The authors would like to thank the anonymous reviewers for valuable comments and constructive suggestions that led to an improved manuscript.

\section{REFERENCES}

[1] Y. Chen, K. B. Letaief, and J. C.-I. Chuang, "Soft-output equalization and TCM for wireless personal communication systems," IEEE J. Select. Areas Commun., vol. 16, pp. 1679-1690, Dec. 1998.
[2] P. Fan, K. B. Letaief, and R. D. Murch, "Analysis of the effects of time delay spread on TCM performance," IEEE J. Select. Areas Commun., vol. 17, pp. 1112-1223, July 1999.

[3] A. J. Paulraj and C. B. Papadias, "Space-time processing for wireless communications," IEEE Signal Processing Mag., vol. 14, pp. 49-83, Nov. 1997.

[4] C. L. Ng, K. B. Letaief, and R. D. Murch, "Antenna diversity combining and finite-tap decision feedback equalization for high-speed data transmission," IEEE J. Select. Areas Commun., vol. 16, pp. 1367-1375, Oct. 1998.

[5] S. L. Ariyavisitakul, J. H. Winters, and I. Lee, "Optimum space-time processors with dispersive interference: Unified analysis and required filter span," IEEE Trans. Commun., vol. 47, pp. 1073-1083, July 1999.

[6] G. J. Foschini and M. J. Gans, "On limits of wireless communications in a fading environment when using multiple antennas," Wireless Personal Commun., vol. 6, pp. 311-335, Mar. 1998.

[7] G. J. Foschini, "Layered space-time architecture for wireless communication in a fading environment when using multi-element antennas," Bell Labs Tech. J., pp. 41-59, Autumn 1996.

[8] V. Tarokh, N. Seshadri, and A. R. Calderbank, "Space-time codes for high data rate wireless communications: Performance criteria and code construction," IEEE Trans. Inform. Theory, vol. 44, pp. 744-765, Mar. 1998.

[9] Y. Gong and K. B. Letaief, "Performance evaluation and analysis of space-time coding in unequalized multipath fading links," IEEE Trans. Commun., vol. 48, pp. 1778-1782, Nov. 2000.

[10] S. M. Alamouti, "A simple transmit diversity technique for wireless communications," IEEE J. Select. Areas Commun., vol. 16, pp. 1451-1458, Oct. 1998.

[11] V. Tarokh, H. Jafarkhani, and A. R. Calderbank, "Space-time block codes from orthogonal designs," IEEE Trans. Inform. Theory, vol. 45, pp. 1456-1467, July 1999.

[12] S. M. Alamouti, V. Tarokh, and P. Poon, "Trellis coded modulation and transmit diversity: Design criteria and performance evaluation," in Proc. IEEE ICUPC'98, Oct. 1998, pp. 703-707.

[13] R. G. Gallager, Information Theory and Reliable Communications. New York: Wiley, 1968.

[14] S. H. Jamali and T. Le-Ngoc, "A new 4-state 8-PSK TCM scheme for fast fading, shadowed mobile radio channels," IEEE Trans. Veh. Technol., vol. 40, pp. 216-222, Feb. 1991.

[15] G. Ungerboeck, "Channel coding with multilevel/phase signals," IEEE Trans. Inform. Theory, vol. 28, pp. 55-67, Jan. 1982. 


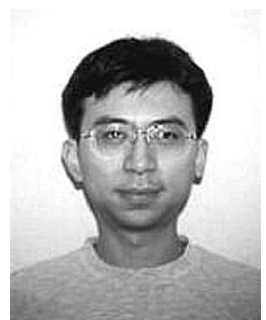

Yi Gong (S'99) received the B.S. and M.S. degrees from Southeast University, Nanjing, China, in 1995 and 1998, respectively, and the Ph.D. degree from the Hong Kong University of Science and Technology (HKUST), Clear Water Bay, Hong Kong, in 2002, all in electrical engineering.

From March 1998 to August 1998, he was a staff member at the Department of Wireless Engineering, Southeast University. Since September 1998, he has been a Research and Teaching Assistant with the Department of Electrical and Electronic Engineering at the Hong Kong University of Science and Technology. His current research interests include space-time coding/processing, OFDM and multicarrier communications, channel estimation and equalization, multiuser communications, and CDMA.

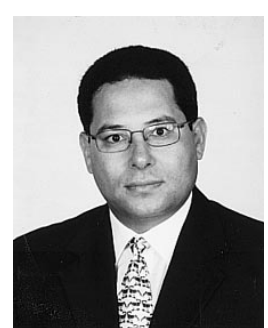

Khaled Ben Letaief (S'85-M'86-SM'97) received the B.S. degree with distinction in electrical engineering from Purdue University, West Lafayette, IN, in December 1984. He received the M.S. and $\mathrm{Ph} . \mathrm{D}$. degrees in electrical engineering from Purdue University, in August 1986 and May 1990, respectively.

From January 1985 and as a Graduate Instructor in the School of Electrical Engineering at Purdue University, he has taught courses in communications and electronics. From 1990 to September 1993, he was a Faculty Member in the EEE Department at the University of Melbourne, Australia where he was also a Member of the Center for Sensor Signal and Information Systems. Since September 1993, he has been with the Electrical and Electronic Engineering Department at the Hong Kong University of Science and Technology (HKUST), Clear Water Bay, Hong Kong, where he is now a Professor. His current research interests include wireless and mobile communications, OFDM, space-time processing for wireless systems, multiuser detection, wireless multimedia communications, and CDMA systems.

Dr. Letaief was appointed the founding Editor-in-Chief of the IEEE TRANSACTIONS ON WIRELESS COMMUNICATIONS, in January 2002. He has served on the Editorial Board of other journals including the IEEE TRANSACTIONS ON COMMUNICATIONS, IEEE Communications Magazine, Wireless Personal Communications and IEEE JouRnal ON SELECTED AREAS In Communications, Wireless Series (as Editor-in-Chief). He served as the Technical Program Chair of the 1998 IEEE GLOBECOM Miniconference on Communications Theory, held in Sydney, Australia. He was also the Co-Chair of the 2001 IEEE ICC Communications Theory Symposium, held in Helsinki, Finland. He is currently serving as Vice-Chair of the IEEE Communications Society Technical Committee on Personal Communications. He is also currently the Vice Chair of Meeting and Conference Committee of the IEEE COMSOC Asia Pacific Board. In addition to his active research activities, he has also been a dedicated teacher committed to excellence in teaching and scholarship. He received the Mangoon Teaching Award from Purdue University in 1990; the Teaching Excellence Appreciation Award by the School of Engineering at HKUST in Spring 1995, Fall 1996, Fall 1997 and Spring 1999; and the Michael G. Gale Medal for Distinguished Teaching (Highest university-wide teaching award and only one recipient/year is honored for his/her contributions). 